Article

\title{
Comparing the Models of Steepest Entropy Ascent Quantum Thermodynamics, Master Equation and the Difference Equation for a Simple Quantum System Interacting with Reservoirs
}

\author{
Charles E. Smith \\ Department of Mechanical Engineering, Virginia Polytechnic Institute and State University, \\ Blacksburg, VA 24060, USA; cesmith@vt.edu; Tel.: +1-540-577-1390 \\ Academic Editors: Umberto Lucia and Giuseppe Grazzini \\ Received: 5 February 2016; Accepted: 4 May 2016; Published: 12 May 2016
}

\begin{abstract}
There is increasing interest concerning the details about how quantum systems interact with their surroundings. A number of methodologies have been used to describe these interactions, including Master Equations (ME) based on a system-plus-reservoir $(\mathrm{S}+\mathrm{R})$ approach, and more recently, Steepest Entropy Ascent Quantum Thermodynamics (SEAQT) which asserts that entropy is a fundamental physical property and that isolated quantum systems that are not at stable equilibrium may spontaneously relax without environmental influences. In this paper, the ME, SEAQT approaches, and a simple linear difference equation (DE) model are compared with each other and experimental data in order to study the behavior of a single trapped ion as it interacts with one or more external heat reservoirs. The comparisons of the models present opportunities for additional study to verify the validity and limitations of these approaches.
\end{abstract}

Keywords: quantum thermodynamics; quantum relaxation; quantum entropy; SEAQT

\section{Introduction}

There is ongoing interest in modeling how quantum systems interact with their environment. The understanding of the relationship between relaxation processes, entropy and quantum mechanics is increasingly considered to be of fundamental importance [1,2]. External and internal influences directly affect many, if not all quantum phenomena, such as quantum computing [3], quantum chemistry [4,5], and entanglement. Methodologies that have been developed to describe quantum dissipative systems are extensive and include stochastic quantum mechanics [6] and nonlinear versions of the Schrödinger Equation [7,8]. Much of the theoretical work describing quantum interactions with the environment has been done by using system-plus-reservoir approaches $(\mathrm{S}+\mathrm{R})$, i.e., Master Equation (ME) techniques $[6,9,10]$. An alternate formalism, Steepest Entropy Ascent Quantum Thermodynamics (SEAQT), developed by Hatsopoulos, Gyftopoulos, and Beretta [11-16], seeks to unify the Second Law of Thermodynamics with Quantum Mechanics. The theory asserts that it is possible for quantum systems as small as one particle to possess entropy, and claims that quantum systems that are not at stable thermodynamic equilibrium may spontaneously relax. SEAQT has been used in previous efforts to model quantum steady state conditions [17].

Single trapped atoms have proved to be an important research tool, and are described extensively in Leibfried et al. [18]. Experimental data relating to the quantum relaxation of single ions include groundbreaking work by Turchette et al. [19] and Gring et al. [20].

The main objective of what follows is to directly compare the ME and SEAQT models as well a simple linear difference equation (DE) to data for interactions of a trapped ion with the environment as presented in [19]. The intent of the comparisons is to reveal similarities, but also differences 
in predictions that can be used to test the validity of the models against the essential but limited experimental data. It is hoped that the results will encourage discussion and help promote further experimentation that further reveals how entropy interrelates with Quantum Mechanics.

\subsection{Model Descriptions}

For this study, the quantum states for the single trapped ion under study are limited to Fock states where off-diagonal elements are equal to zero. Fock states were chosen because they are simpler and easier to handle computationally for the SEAQT cases. Nevertheless, they represent legitimate quantum states of interest for which experimental data is available. Fock states do not, however, exhibit behavior such as interference effects and quantum entanglement.

Important theoretical work using the traditional ME $(S+R)$ approach has been done by $[21,22]$ for trapped ions interacting with a heat bath. The ME model is said to be valid for systems that are near or far from stable equilibrium [21]. The ME model assumes that the system-reservoir coupling is relatively weak so that the system maintains eigenlevels that are unaltered by the reservoir. Though the ME model is based on the reversible Schrödinger Equation, irreversibility is achieved by various approximations $[6,9,10]$. A straightforward presentation is found in [19] for density matrices with or without non-zero off-diagonal elements.

The Master Equation for a single particle interacting with a reservoir is expressed as

$$
\frac{d \rho}{d t}=\frac{\gamma}{2}(\bar{n}+1)\left(2 \hat{a} \rho \hat{a}^{+}-\hat{a}^{+} \hat{a} \rho-\rho \hat{a}^{+} \hat{a}\right)+\frac{\gamma}{2} \bar{n}\left(2 \hat{a}^{+} \rho \hat{a}-\hat{a} \hat{a}^{+} \rho-\rho \hat{a} \hat{a}^{+}\right)
$$

where $\rho$ is the state matrix, $\hat{a}$ is the lowering operator, and $\gamma$ is the system decay rate or reciprocal time constant. The dynamics of the reservoir are not contained explicitly in Equation (1), other than the quantity $\bar{n}$, which represents the average energy of the reservoir as it interacts with the system. The energy is in terms of the number of system quanta $\hbar \omega_{0}$ and is related to the reservoir temperature by:

$$
\bar{n}=\left(\frac{e^{-\hbar \omega_{0} / k_{B} T}}{1-e^{-\hbar \omega_{0} / k_{B} T}}\right) .
$$

The solution to the Master Equation for Fock state evolution is shown in $[19,21]$ and takes the form

$$
\rho_{n n}(t)=\frac{1}{1+N(t)} \sum_{j=0}^{n}\left(\frac{N(t)}{1+N(t)}\right)^{j}\left(\frac{e^{-\gamma t / 2}}{1+N(t)}\right)^{2 n-2 j} \times \sum_{j=0}^{\infty}\left(1-\frac{e^{-\gamma t}}{1+N(t)}\right)^{j}\left(\begin{array}{c}
n+1-j \\
n-j
\end{array}\right) \times\left(\begin{array}{c}
n \\
j
\end{array}\right) \rho_{n+l-j, n+l-j}(0)
$$

where $N(t)$ is the average number of quanta in the system over time for a system initially in the ground state:

$$
N(t)=\bar{n}\left(1-e^{-\gamma t}\right) .
$$

The index $n$ stands for the nth energy eigenlevel above the groundstate.

The difference equation DE is a simple dynamic relaxation model that can be constructed based on the difference between the current state of the system and the final mutually stable equilibrium state with the reservoir [23,24]. It is understood that the region of greatest accuracy occurs in the "linear" region close to stable equilibrium, which may include steady state behavior. The model has the advantage of being simple enough to readily approximate several interacting reservoirs. The governing equation is,

$$
\frac{d \rho_{n n}(t)}{d t}=-\frac{1}{\tau}\left(\rho_{n n}(t)-\rho_{\text {res }}\right)
$$

where $\tau$ is a time constant. The state of the reservoir is assumed to be constant in time.

The SEAQT formalism is based on the idea that entropy is a fundamental property of matter that is present for all systems, even those at quantum scales. The theory asserts that the kinematical framework for entropy as a fundamental quantity is established by the state matrix $\rho$ of quantum 
mechanics, which is fully able to describe entropy for a quantum system as well as other quantities, such as energy and momentum. The formalism asserts that any system that is not at stable thermodynamic equilibrium, even a system of one particle, is by default unstable and will tend to spontaneously relax toward the equilibrium state [25]. SEAQT postulates that the evolution takes place along a path of steepest entropy increase [26]. The change occurs due to the ability of the internal degrees of freedom; i.e., the energy eigenlevels of the system, to exchange energy with one another [27]. Relaxation may however be inhibited by the presence of local metastable states, such as those robust enough to describe chemistry and the existence of atomic elements.

To enable entropy changes to occur, SEAQT adds a nonlinear operator to the time-dependent Schrödinger Equation, which produces an expression analogous to a master equation [24]. The SEAQT evolution equation claims to describe the evolution of all systems whether they are near or far from stable equilibrium. Recently, SEAQT has been extended and applied to quantum chemistry [28], fuel cells [17], and quantum decoherence [29].

The SEAQT formalism also exhibits a time lag effect that is not present with the other two approaches. When a system is near a pure state close to zero entropy or a "partial equilibrium state" [27], the energy is contained in only a few of the available energy eigenlevels. Relaxation proceeds slowly until the energy becomes more evenly distributed. The dynamics of the SEAQT model are given by the equation developed by Beretta [15]:

$$
\frac{d \rho}{d t}=-\frac{i}{\hbar}[H, \rho]-\frac{1}{\tau} D(\rho(t))
$$

The term in brackets is the time-dependent Schrödinger term, along with $D$, a nonlinear irreversibility operator. Equation (6) describes the time evolution of the state of an isolated, non-equilibrium quantum system as it relaxes spontaneously to a stable equilibrium end state along a path of steepest entropy increase. If the system is in a Fock state, Equation (6) simplifies to

$$
\frac{d \rho}{d t}=-\frac{1}{\tau} D(\rho(t))
$$

The irreversibility operator for the $n$-th energy eigenlevel can be represented by a ratio of Gram determinants [25]:

$$
D_{n}=\frac{\left|\begin{array}{ccc}
\rho_{n} \ln \rho_{n} & \rho_{n} & e_{n} \rho_{n} \\
\sum \rho_{i} \ln \rho_{i} & 1 & \sum e_{i} \rho_{i} \\
\sum e_{i} \rho_{i} \ln \rho_{i} & \sum e_{i} \rho_{i} & \sum e_{i}^{2} \rho_{i}
\end{array}\right|}{\left|\begin{array}{cc}
1 & \sum e_{i} \rho_{i} \\
\sum e_{i} \rho_{i} & \sum e_{i}^{2} \rho_{i}
\end{array}\right|}
$$

\subsection{Equations for Describing System Interactions}

New expressions were developed herein to describe the interactions between the elements of composite systems when employing the SEAQT and DE approaches. Several cases are illustrated in Figure 1. In Figure 1a, two systems relax spontaneously and independently without mutual interaction. A relation that combines the two separate equations of motion for uncorrelated systems $\rho_{A}$ and $\rho_{B}$ into a single equation for a composite system is shown in [24]. The separate equations for systems $A$ and $B$ are

$$
\frac{d \rho_{A}}{d t}=\frac{-i}{\hbar}\left[H, \rho_{A}\right]-\frac{1}{\tau_{A}} D\left(\rho_{A}\right)
$$

and

$$
\frac{d \rho_{B}}{d t}=\frac{-i}{\hbar}\left[H, \rho_{B}\right]-\frac{1}{\tau_{B}} D\left(\rho_{B}\right)
$$


The state matrices of the two systems can be combined using an outer product. The equivalent expression for the composite system becomes

$$
\frac{d\left(\rho_{A} \otimes \rho_{B}\right)}{d t}=\frac{-i}{\hbar}\left[H, \rho_{A} \otimes \rho_{B}\right]-\frac{1}{\tau_{A}} D\left(\rho_{A}\right) \otimes \rho_{B}-\frac{1}{\tau_{B}} D\left(\rho_{B}\right) \otimes \rho_{A},
$$

a form that is reminiscent of the product rule for differentiation. Equation (11) can be expressed in words as "the change with time of the composite of $A$ and $B$ is equal to the Schrödinger term for the composite, minus the spontaneous relaxation of $A$ without the influence of $B$, minus the spontaneous relaxation of $B$ without the influence of $A$." The speeds of relaxation for $A$ and $B$ are governed by the time constants $\tau_{A}$ and $\tau_{B}$. Though expressed as a composite, the two systems remain uncorrelated and their relaxations take place independently.

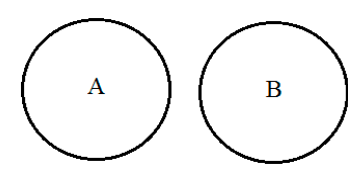

a)

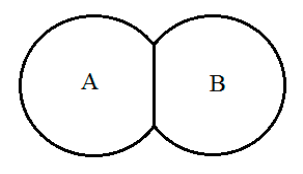

b)
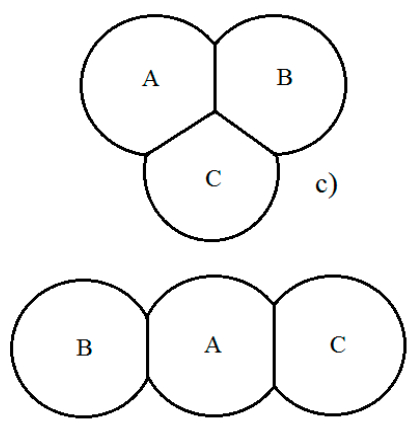

d)

Figure 1. Representations of composite system interactions. (a) Two systems evolve spontaneously and independently; (b) Two systems evolve spontaneously as they also interact; (c) Three systems that interact. Each region represents a separate spontaneous evolution with its own time constant. Each interface or intersection of interfaces represents a mutual interaction of two or more systems that also has its own time constant; (d) Interactions of system $A$ with reservoirs $B$ and $C$, which may include steady state behavior.

To enable the interaction between systems $A$ and $B$, an extension is introduced to allow the composite $\rho_{A} \otimes \rho_{B}$ to evolve under the influence of the irreversibility operator. The resulting term, $-\frac{1}{\tau_{A B}} D\left(\rho_{A} \otimes \rho_{B}\right)$, is added to Equation (11), where $\tau_{A B}$ is a separate time constant for the interaction. For convenience, $D\left(\rho_{A} \otimes \rho_{B}\right)$ is rewritten as $D\left(\rho_{A B}\right)$ and can be expressed as "the relaxation of the composite of $A$ and $B$ due to the interaction of $A$ with $B^{\prime \prime}$. If the state matrices $\rho_{A}$ and $\rho_{B}$ have off-diagonal terms equal to zero, the Schrödinger term can be dropped. The equation for case (b) in Figure 1 is then

$$
\frac{d\left(\rho_{A B}\right)}{d t}=-\frac{1}{\tau_{A}} D\left(\rho_{A}\right) \otimes \rho_{B}-\frac{1}{\tau_{B}} D\left(\rho_{B}\right) \otimes \rho_{A}-\frac{1}{\tau_{A B}} D\left(\rho_{A B}\right) .
$$

Given that system $B$ is a reservoir (already at equilibrium, off-diagonal terms are zero), Equation (12) reduces to

$$
\frac{d\left(\rho_{A B}\right)}{d t}=-\frac{1}{\tau_{A}} D\left(\rho_{A}\right) \otimes \rho_{B}-\frac{1}{\tau_{A B}} D\left(\rho_{A B}\right) .
$$

If the spontaneous relaxation of $A$ is slow, the first term on the right hand side of the equation can also be dropped. 
For the case of three systems $A, B, C$, that interact with one another as shown in case (c), the above formalism for a Fock state is written as:

$$
\begin{aligned}
& \frac{d\left(\rho_{A B C}\right)}{d t}=-\frac{1}{\tau_{A}} D\left(\rho_{A}\right) \otimes \rho_{B} \otimes \rho_{C}-\frac{1}{\tau_{B}} D\left(\rho_{B}\right) \otimes \rho_{A} \otimes \rho_{C}-\frac{1}{\tau_{C}} D\left(\rho_{C}\right) \otimes \rho_{A} \otimes \rho_{B} \\
& -\frac{1}{\tau_{A B}} D\left(\rho_{A B}\right) \otimes \rho_{C}-\frac{1}{\tau_{A C}} D\left(\rho_{A C}\right) \otimes \rho_{B}-\frac{1}{\tau_{B C}} D\left(\rho_{B C}\right) \otimes \rho_{A}-\frac{1}{\tau_{A B C}} D\left(\rho_{A B C}\right) .
\end{aligned}
$$

Each spontaneous relaxation and relaxation due to interaction is distinct and has its own time constant.

For case (d), where a small system $A$ in a Fock state interacts with two reservoirs $B$ and $C$, Equation (14) becomes,

$$
\frac{d\left(\rho_{A B C}\right)}{d t}=-\frac{1}{\tau_{A}} D\left(\rho_{A}\right) \otimes \rho_{B} \otimes \rho_{C}-\frac{1}{\tau_{A B}} D\left(\rho_{A B}\right) \otimes \rho_{C}-\frac{1}{\tau_{A C}} D\left(\rho_{A C}\right) \otimes \rho_{B} .
$$

The two reservoirs interact only with system $A$ and not with each other. Again, the self-relaxation term for system $A$ can be ignored if it is deemed slow enough.

The proposed interaction term, $-\frac{1}{\tau_{A B}} D\left(\rho_{A B}\right)$ is simplistic and may not fully describe all interaction scenarios that physically occur. The term represents a composite of two or more uncorrelated systems, where the irreversibility operator has joint and unimpeded influence on the degrees of freedom of each as the evolution takes place. Though simplistic, the interaction term fits into the dynamic framework of Equation (6) as developed by Beretta: The combined system is itself an isolated system that spontaneously evolves to a maximal entropy state along a steepest entropy path. The interaction term therefore represents a valid case within the SEAQT theory. The extended formalism represented by Equations (12)-(15) allows for the separate relaxation of each subsystem to be included as well.

\subsection{Additional Modeling Details}

The model system under investigation is a single trapped ion held in a quadratic potential that is able to move along one dimension, similar to experiments involving a Paul trap [18]. The system behaves as a quantum harmonic oscillator with equally-spaced energy eigenlevels. The reservoir with which the system interacts is modeled as a bath of 3-dimensional quantum oscillators having a degeneracy of

$$
g=\frac{(n+1)(n+2)}{2}
$$

where $n$ is the $n$-th eigenenergy level of the oscillator.

Initially, all interacting systems are regarded as independent and uncorrelated. An initial state for each system (i.e., $\rho(0))$ is chosen. The systems are closed, with no mass flow across their boundaries, but they may exchange energy and entropy via heat interactions. For the SEAQT case, the system and reservoir are combined and the evolution for the composite is calculated. Afterward, the evolution of the system of interest is extracted from the composite results using a partial trace operation.

\subsection{Treatment of Reservoirs}

The ME and DE have been formulated to describe the evolution of the system of interest without including explicit details of the reservoir. The system of interest moves to a final stable equilibrium state that has the same temperature as the reservoir. The ME interaction scenarios were determined directly from Equation (3). For DE, Equation (5) was used. A solution for interactions with more than one reservoir using the ME approach has yet to be developed, but is being pursued by [30].

The SEAQT technique was implemented with a reservoir that was explicitly modeled as a bath of a number of harmonic oscillators. The bath remains in a stable equilibrium state relative to itself; i.e., in a thermal state, but is free to exchange energy and entropy with the system of interest. Though finite in size, the bath of oscillators was large enough to approximate the effects of a system approaching that of a reservoir for the lowest energy eigenlevels. 


\section{Results and Discussion}

Figures 2-5 show comparisons between the ME, DE, and SEAQT models for the case where the single-particle system near a pure state interacts with a "cold" reservoir at lower energy. Initial conditions for the three cases are identical. Spontaneous self-relaxation for the DE and SEAQT cases are excluded, except in Figure 5 for SEAQT. Given the lack of data for this interaction, the respective time constants were adjusted to produce energy $v s$. time curves having the same shape in order to facilitate comparisons between the models.

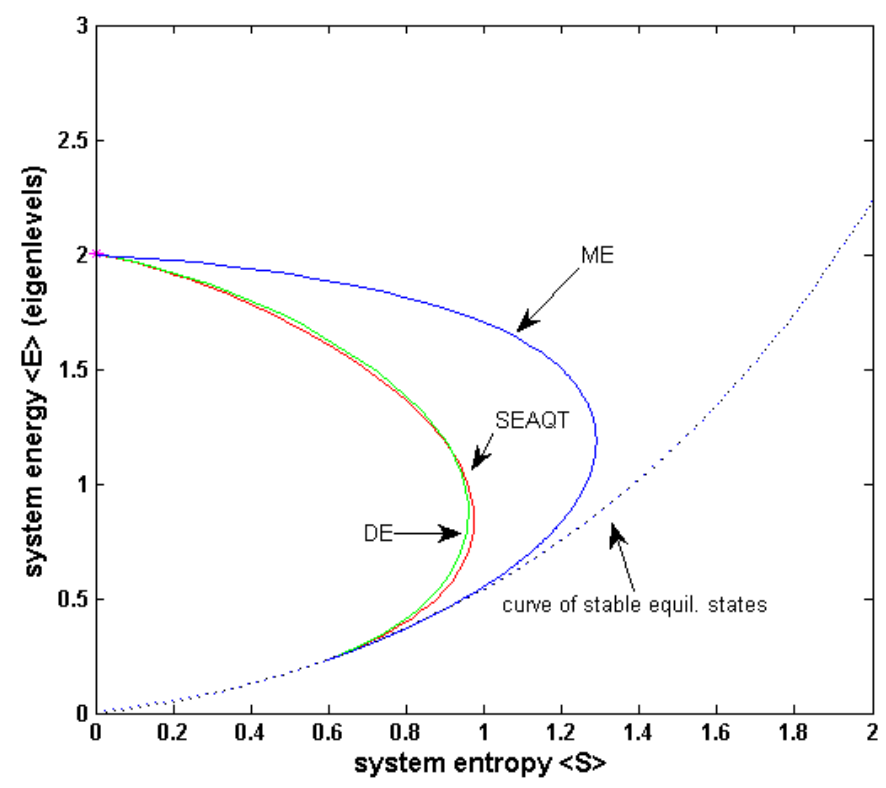

Figure 2. Comparison of Energy vs. Entropy trajectories using the master equation (ME), difference equation (DE), and steepest entropy ascent quantum thermodynamics (SEAQT) models for a single particle system as it interacts with a "cold" reservoir. Relaxation occurs only via interaction with the reservoir. Spontaneous relaxation effects are excluded. The initial state is near eigenenergy of 2. The final state is at stable equilibrium where the slope corresponds to the temperature of the reservoir.
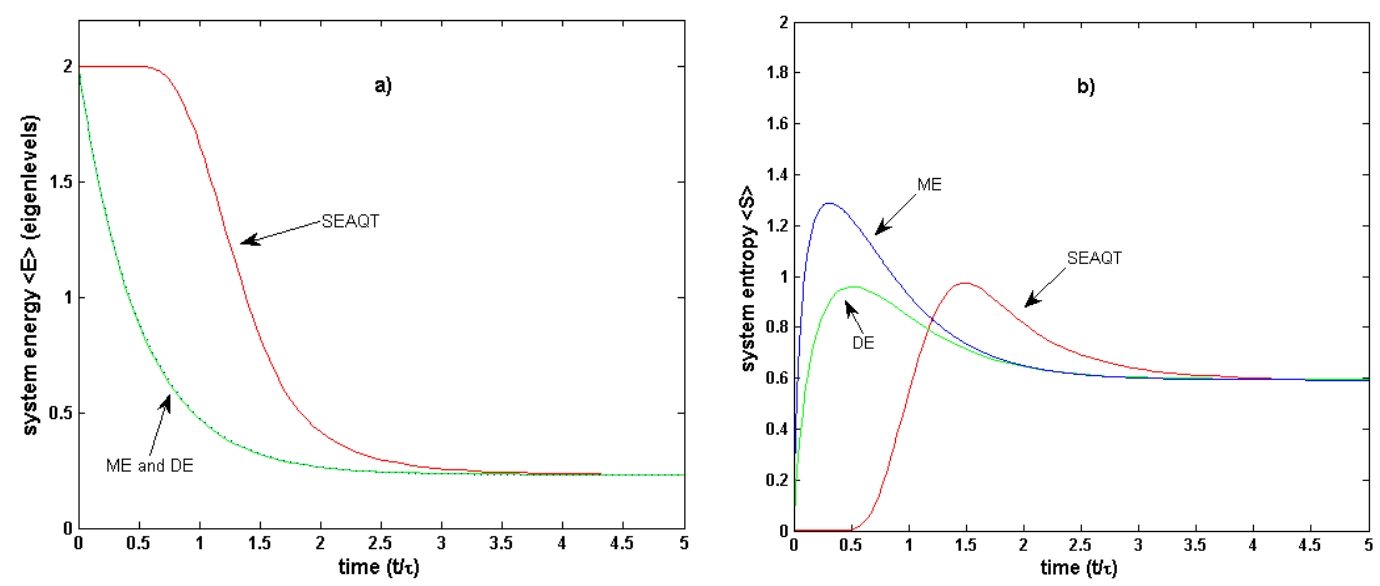

Figure 3. Comparisons of time trajectories for the ME, DE, and SEAQT models for the case in Figure 2. (a) For the energy vs. time comparison, the paths for the ME and DE are essentially identical. The trajectory for the SEAQT model is also similar, except for the time lag, which is due to the initial slowness of energy spreading between eigenlevels; (b) For the entropy vs. time trajectories, the ME model shows a markedly greater change in entropy. The DE and SEAQT curves indicate similar dynamic behavior. 


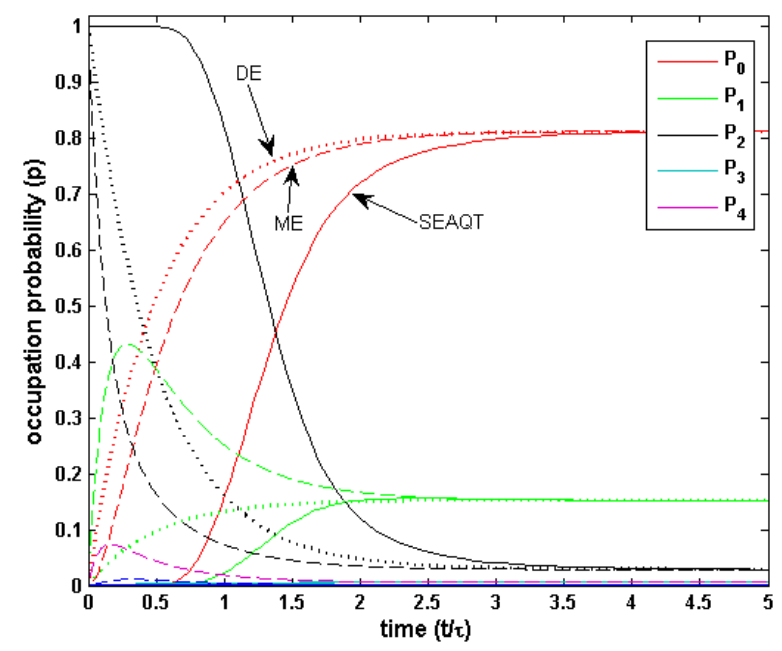

Figure 4. Evolution $v$ s. time of the state matrix $\rho(t)$ for the lowest five energy eigenlevels for the system interaction with the "cold" reservoir of Figure 2. The various colors represent the different energy eigenlevels of the system. The dotted lines represent the results for the DE model, the dashed lines represent the ME model, and the solid lines are for the SEAQT model. The occupation probabilities for P1 and P4 change the fastest and over the widest ranges with the ME model. An example of the characteristic time delay for the SEAQT case is seen.

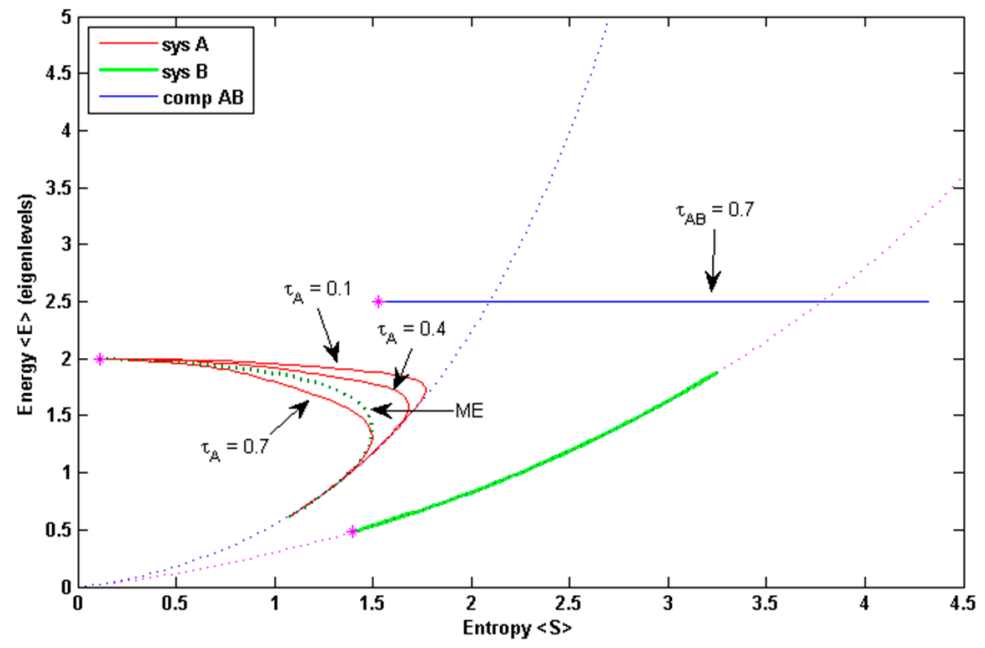

Figure 5. Energy vs. Entropy curves of SEAQT as described by Equation (13) for the system of interest $A$ (shown in red) which includes spontaneous relaxation effects of various magnitudes as well as interaction with the reservoir. The evolution of reservoir $B$ is in green, and the composite system $A \otimes B$ is in blue. Magenta asterisks mark the initial states. An ME curve is shown for comparison.

In Figure 2, the $E-S$ trajectory of the SEAQT model closely resembles that for the linear DE, and was found to become more coincident as the size of model reservoir for SEAQT is increased. Both paths markedly diverge from the predictions of ME. The differences may be due to the simplistic nature of the interaction model used for the SEAQT case. However, near-linear behavior by the SEAQT model has been seen previously. A straightforward examination of the results for isolated systems in [31] shows trajectories that are almost indistinguishable from simple decaying exponentials of the DE model. In Figure 2 and other figures, the units of entropy are (eigen) energy units/K with the value of the Boltzmann constant set to unity.

The energy vs. time diagram in Figure 3a shows essentially identical behavior for ME and DE. The SEAQT trajectory displays a time lag, but behaves similarly to ME and DE at later times. There is 
limited literature illustrating the time lag that is predicted by SEAQT. The results found by [32] for the relaxation of rubidium atoms hints at a time delay with a sigmoidal profile and can be contrasted to that of [33,34]. The data of Figure 15 in Turchette et al. [19] displays what looks like time delays for at least two of the cases studied.

Figure $3 \mathrm{~b}$ shows that the entropy increases faster and to a greater extent for the ME model than either DE or SEAQT. Figure 4 illustrates how the occupation probabilities for the elements of the state matrices for the three models change with time.

The $E-S$ curves in Figure 5 show how discrepancies between the SEAQT and ME are reduced when spontaneous relaxation effects are included according to Equation (13) for system $A$, the system of interest. However, the curvature for the SEAQT model appears less rounded than that for the ME model over a range of time constants.

The SEAQT formalism does however allow for $\tau$ to be a functional that varies with the system state [24], so that

$$
\tau=\tau(\rho)
$$

Changing the value of $\tau_{A B}$ alone changes the rate of the system evolution but not the shape of the $E-S$ curve. By appropriately varying $\tau(\rho)$ for both spontaneous and reservoir interaction effects, the $E-S$ curve for SEAQT or DE could be made to conform to the ME trajectory. However, at present, there is not yet a rationale for choosing how time factors should vary, other than to avoid conflict with the Heisenberg uncertainty [24].

Figure 5 also shows the $E-S$ curves for the composite system as well as the finite reservoir. The curves illustrate how energy and entropy are transferred between system A and the reservoir. As expected, energy is conserved for the composite system and entropy increases overall due to the irreversibilities of the relaxation.

Current experimental evidence indicates that spontaneous relaxation effects for single particle systems, if they exist, are negligible. Atoms that are sufficiently isolated have been found to maintain their prepared states without degradation for long periods; in the case of rubidium atoms, for more than one minute [34], and for trapped ions, more than ten minutes [35].

In Figures 6 and 7 the comparative behavior for system interactions with a "hot" reservoir are shown. The same general differences between ME, DE, and SEAQT are seen. A closer match with ME can be obtained if spontaneous relaxation effects are included for the SEAQT model.
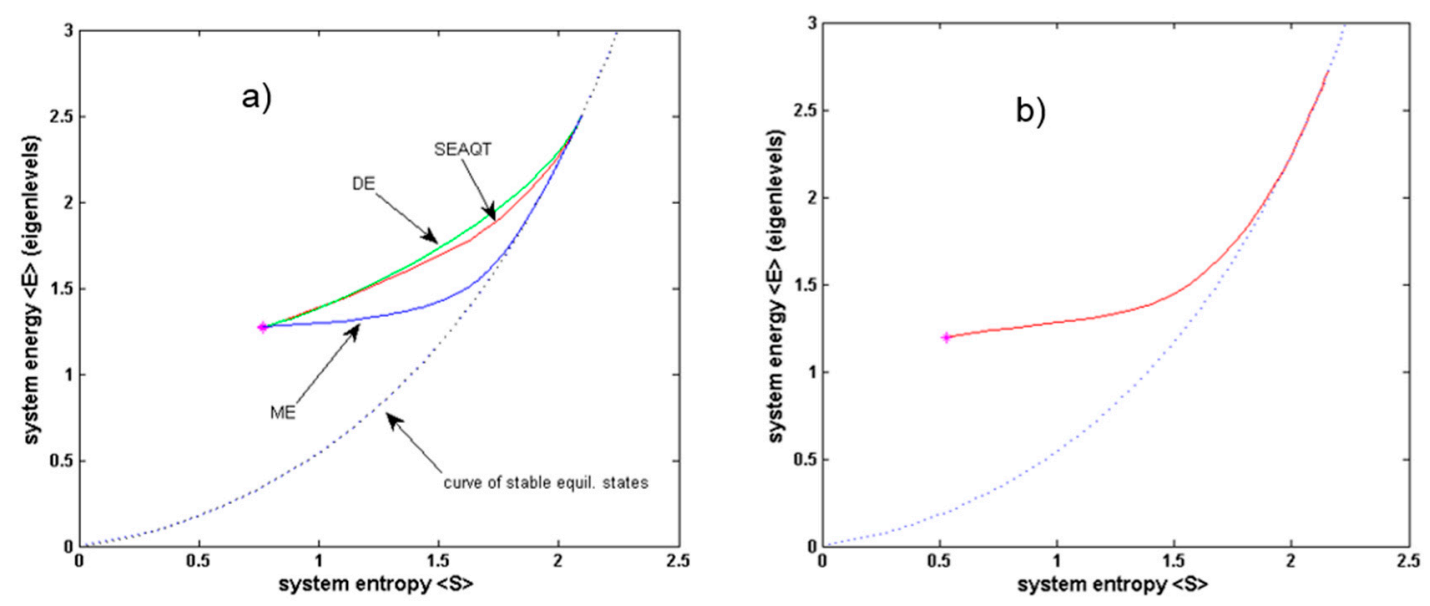

Figure 6. Comparison of Energy vs. Entropy trajectories using the ME, DE, and SEAQT models for a single particle system interacting with a "hot" reservoir. Spontaneous relaxation effects are excluded in (a); but included for the SEAQT case in (b). A magenta asterisk marks the initial state. 


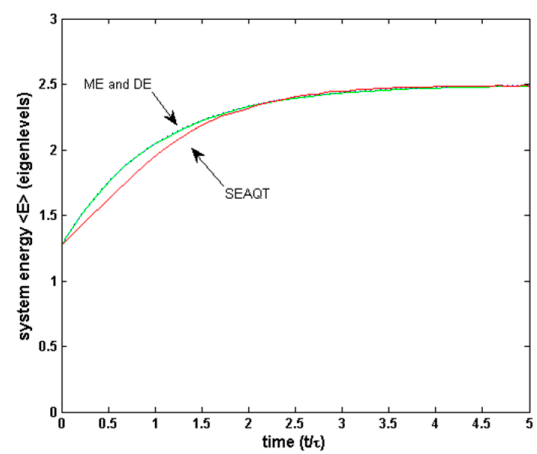

(a)

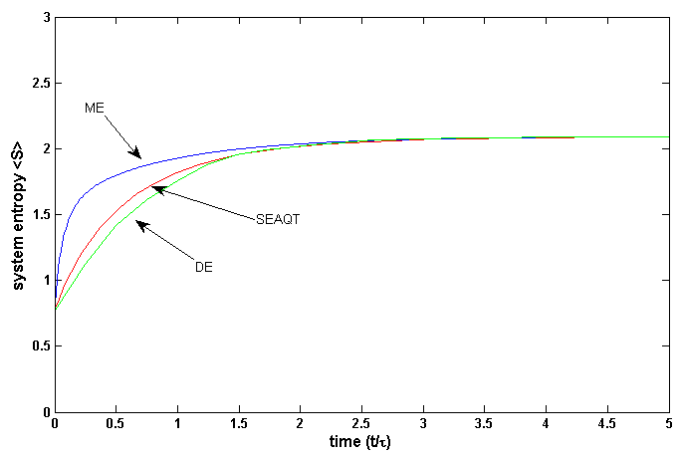

(b)

Figure 7. Energy and entropy time trajectories for the ME, DE, and SEAQT models for a system interacting with a "hot" reservoir as in Figure 6a, without spontaneous relaxation by the system. As in Figure 2, the DE and SEAQT paths tend to more closely coincide as the size of the SEAQT model reservoir is increased. In (a), energy changes vs. time are shown; In (b), the ME model predicts markedly faster changes in entropy with time.

\subsection{Comparison of the Models with Experimental Data}

One of the few sources of experimental data that are available for the relaxation of a quantum single particle system is found in the work of Turchette et al. [19]. The data for the relaxation of Fock states as they interact with a surrogate reservoir are shown therein in Figure 15 for the lowest five energy levels. Four different cases are presented, representing initial states that are prepared near the ground $(|0\rangle)$, first $(|1\rangle)$, second $(|2\rangle)$, and third $(|3\rangle)$ energy eigenstates, respectively. The thermal reservoir was created by introducing a Gaussian noise spectrum to the electromagnetic fields that trap the atom. The evolution of the system state over time was achieved by varying the power of the reservoir over a constant time interval rather than by keeping the reservoir power constant and making measurements over different time intervals. Maintaining a constant time interval has the advantage of limiting extraneous environmental effects not represented by the reservoir. It is not clear whether the higher power levels of the reservoir might cause unintended changes to the eigenstructure of the system, however. Each data point is an average of several thousand experimental runs.

Figure A1 in the Appendix shows the application of Equation (3) of the ME model to the data. The claim in [19] is that the variation of only one parameter, the inverse time constant $\gamma$, is needed to fit the theoretical curves. In practice, the values for the data near the initial state are open to interpretation and may be modified slightly to adjust the curve fit. Moreover, the increase of the mean energy chosen for the reservoir can cause the curves to group more tightly together as equilibrium is approached. The energy for the reservoir in [19] is not known, but the mean reservoir energy for the present study was chosen to be 25 system eigenenergy units. The overall ME data match appears to be reasonably accurate. The results of the correlation with the evolution for state $\mid 1>$ ostensibly shows the worst fit, with traces $\mathrm{P}_{0}, \mathrm{P}_{1}$, and $\mathrm{P}_{2}$ consistently separated from the data points.

Small but noticeable differences are also seen when the current ME results are compared to Figure 15 of [19]. The probabilities for the lowest five theoretical traces sum to greater than unity in the areas near the vertical axis, for example. Other slight shifts of the theoretical curves are seen to imply time delays in the evolution of certain energy levels. It was found in the present study, however, that Equation (3) by itself is not able to produce time lags, regardless of the initial state of the system. Time lags, if they exist, are noteworthy, and would support the SEAQT point of view.

In Figure 8, the SEAQT model utilizing Equation (13) with spontaneous effects is fitted to the data from [19] for the lowest four of the initial states. For the horizontal scale, time is equivalenced to reservoir power as was done in [19]. Reasonable correspondence to data can be made even though the results differ somewhat from the ME result. For the individual cases illustrated, both $\tau_{A}$ and $\tau_{A B}$ were held constant. Unlike the ME and DE models, SEAQT is readily able to produce time lags as seen in 
the case of $\mid 1>$ of Figure 8. Reducing the initial probability for a given energy eigenlevel produces a longer time delay in its corresponding probability curve.

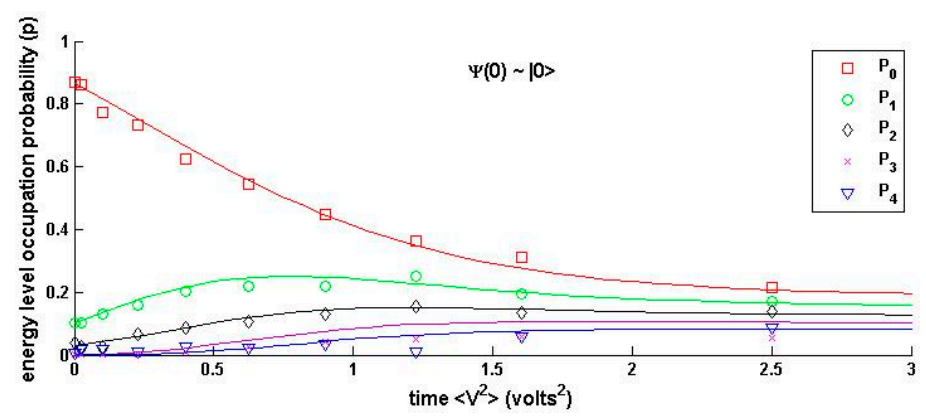

(a)

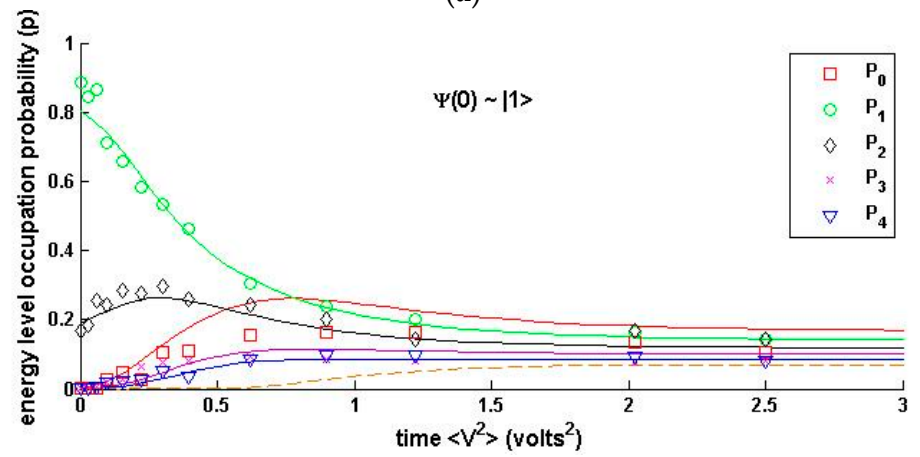

(b)

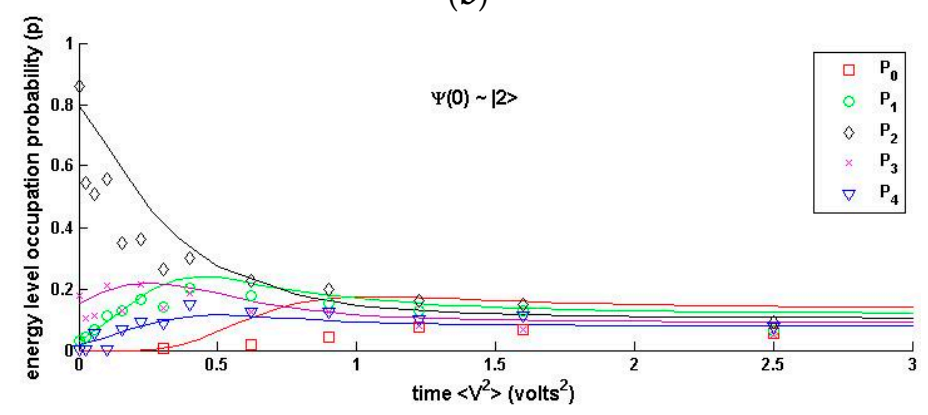

(c)

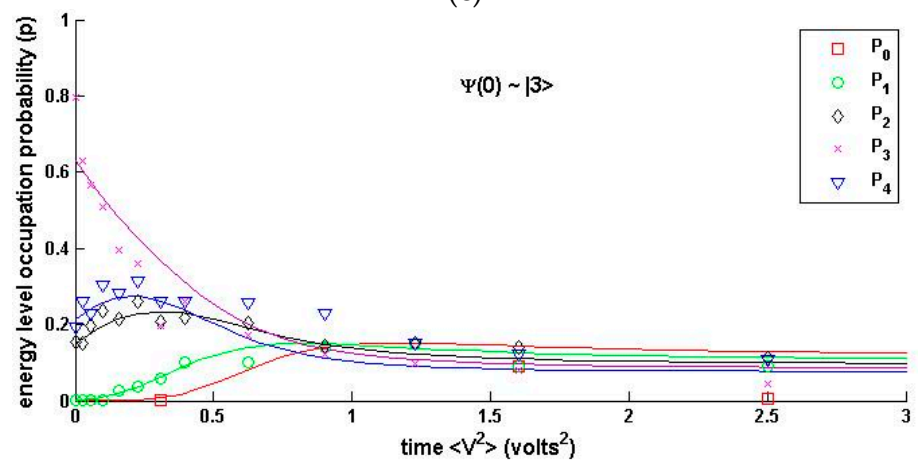

(d)

Figure 8. Comparison of the SEAQT theoretical curves with spontaneous relaxation effects to the experimental data of [19] for initial states approximating $|0\rangle(\mathbf{a}),|1\rangle(\mathbf{b}),|2\rangle(\mathbf{c})$, and $|3\rangle(\mathbf{d})$ based on Equation (13). The dashed tan line for $\mathrm{P}_{5}$ of $|1\rangle$ (b) illustrates a time lag that can be introduced using the SEAQT formalism by making the initial probability small, in this case $1 \times 10^{-5}$. For case $|2\rangle$ (c), there appears to be a time delay in the data for $\mathrm{P}_{0}$. The theoretical curve rises much faster than the data, however. 
Unexpectedly, even when spontaneous effects are neglected for the SEAQT model, there is still reasonable correspondence to the data, as seen in Figure 9. The ME model looks to be slightly if not conclusively better at matching the two higher energy data sets $|2\rangle$ and $|3\rangle$. Figure 10 is an example showing that the DE model without spontaneous effects is not able to adequately capture the inflections of the experimental data.

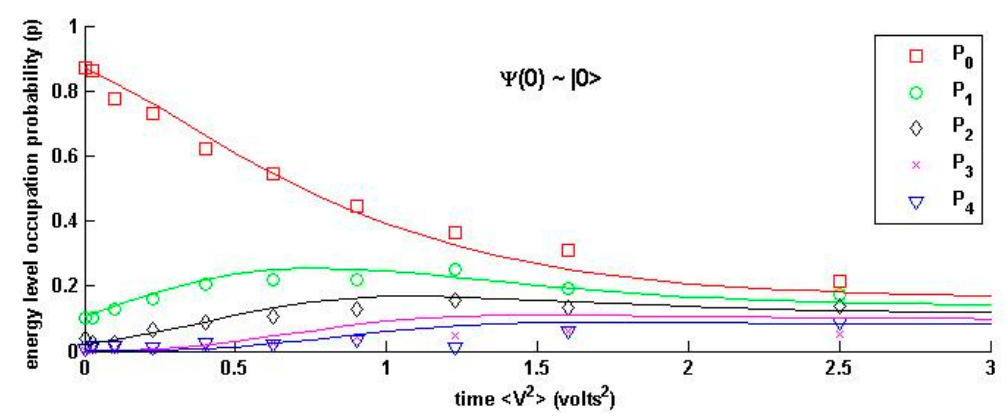

(a)

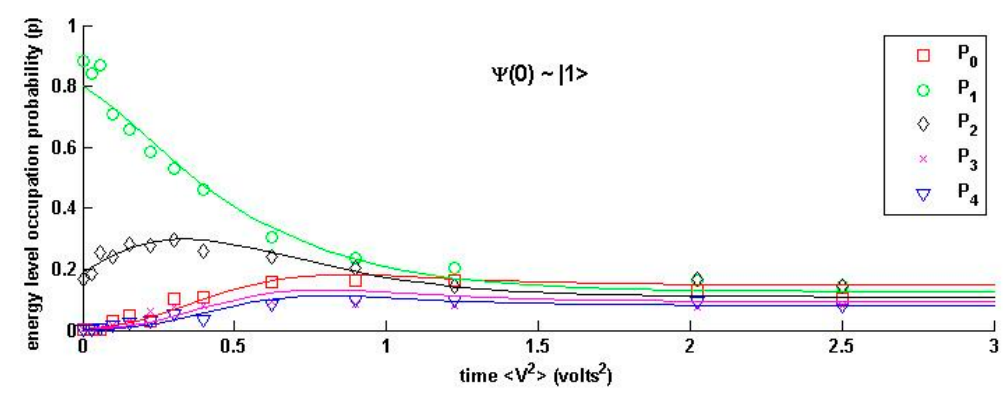

(b)

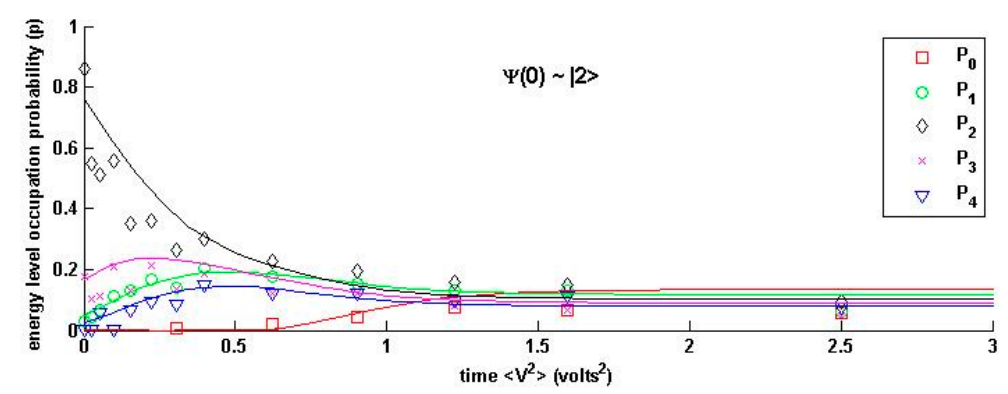

(c)

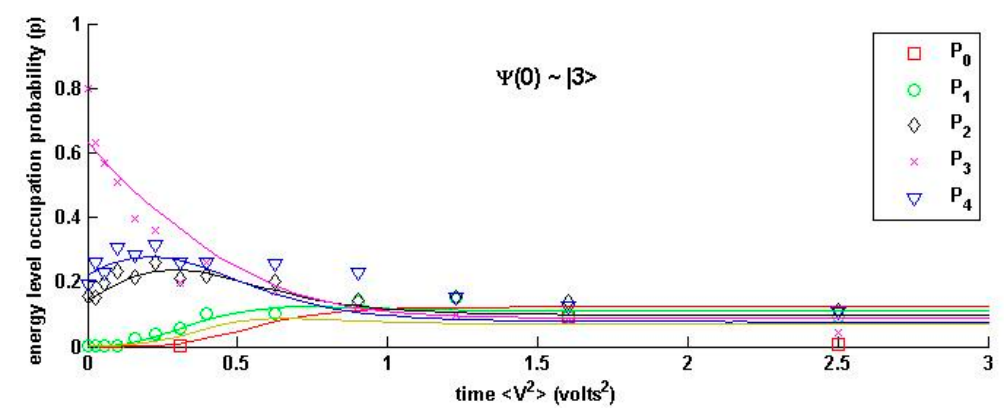

(d)

Figure 9. SEAQT theoretical curves without spontaneous relaxation effects, (a), (b), (c), (d) for initial states $|0\rangle,|1\rangle,|2\rangle,|3\rangle$ as fitted to the data from [19]. The correlation of the curves with the data appears comparable to ME as well as SEAQT with relaxation effects. 


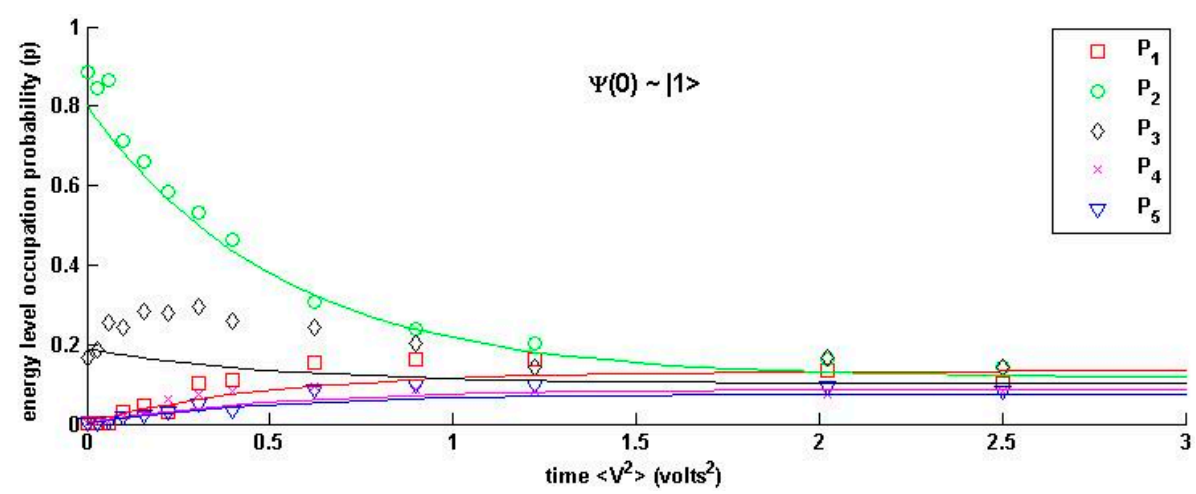

Figure 10. A result for the DE model without spontaneous relaxation effects as compared to the data for state $\mid 1>$. Though accurate in many respects, the DE model does not capture local maxima (e.g., $\mathrm{P}_{3}$ ) in the data.

To best fit the data, it was found necessary to use different time constants for each of the initial states for both the ME and SEAQT models. The implication is that $\tau$ and $\gamma$ are not constant but vary as the system state changes. The values of $\tau$ that were used for each initial state are shown in Table 1 . The ME model evolved more quickly the greater the difference in energy between the initial and final (reservoir) equilibrium state was. Since the reservoir energy was held constant, perhaps the smaller energy differences explain the larger time constants for the higher energy $|2\rangle$ and $|3\rangle$ initial states. For the SEAQT cases with spontaneous effects, the same general trend for $\tau_{A B}$ was seen. Curiously, the opposite tendency was found for the SEAQT cases without spontaneous effects.

Table 1. A table showing how the time constants values were varied in order to fit the ME and SEAQT models to the experimental data. The second column are $\tau$ values for ME, the third and fourth are for SEAQT with spontaneous relaxation, and the last column for SEAQT without spontaneous relaxation effects.

\begin{tabular}{ccccc}
\hline Initial State & $\tau_{M E}(=\mathbf{1} / \gamma)$ & $\tau_{A B}$ & $\tau_{A}$ & $\tau_{A B}$ (No Spontaneous) \\
\hline $\mid 0>$ & 2.1 & 0.40 & 0.40 & 0.60 \\
$\mid 1>$ & 3.0 & 0.50 & 0.40 & 0.32 \\
$\mid 2>$ & 3.1 & 0.55 & 0.45 & 0.25 \\
$\mid 3>$ & 3.7 & 0.65 & 0.45 & 0.26 \\
\hline
\end{tabular}

\subsection{Quantum Steady States}

Figure 11 shows an evolution of a single-atom system that interacts with two reservoirs at different temperatures. The dynamics are based on a DE model using Equation (15). The system is seen to move to a steady state condition that is not at stable equilibrium. The starting points are a pure state with near-zero entropy and a representative stable equilibrium state. The results are considered accurate only in the region near stable equilibrium. The model demonstrates at least qualitatively how the system can evolve to a steady state. Each point on the steady state curve is a linear combination of the two reservoir states. The location of a steady state point is determined by the relative influence of each reservoir on the system as given by the magnitudes of the time constants for the system-reservoir interactions:

$$
\rho_{s s A}=\alpha \rho_{e q A}\left(T_{B}\right)+(1-\alpha) \rho_{e q A}\left(T_{C}\right) ; \quad 0<\alpha<1
$$

where

$$
\alpha=\frac{\tau_{A C}}{\tau_{A B}+\tau_{A C}}
$$




$$
1-\alpha=\frac{\tau_{A B}}{\tau_{A B}+\tau_{A C}} .
$$

Ordinarily, a state with a given energy and entropy that is not at stable equilibrium can be represented by an unlimited number of different distinct state matrices. However, since the stable equilibrium states are unique $[36,37]$, it can be inferred that the steady states are also unique for a given value of the $\alpha$ parameter.

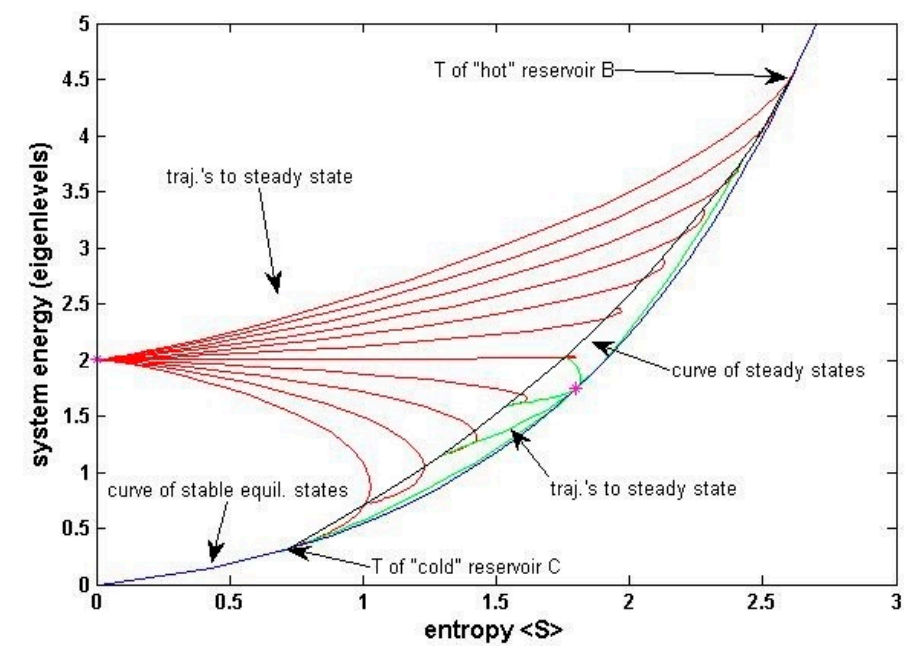

Figure 11. Evolution to steady state predicted by DE of a single atom system as it interacts with two reservoirs at different temperatures. One of the starting states is near a pure state with energy of 2 (red trajectories), while the other is at a stable equilibrium state (green trajectories). The reservoir temperatures correspond to the slopes of the stable equilibrium curve at the indicated locations.

\section{Conclusions}

Comparisons between the ME, DE, and SEAQT methods for modeling the quantum evolution of a single trapped particle that interacts with a reservoir have been presented. A simple, novel formalism for describing interactions between two or more systems has also been presented and applied to the DE and SEAQT approaches. A straightforward adaptation of the DE model was used to demonstrate steady state dynamics for a single particle system that interacts with two reservoirs at different temperatures.

Both the ME and SEAQT approaches are shown to reasonably coincide with the existing experimental data. The SEAQT model matches the data, even without the addition of spontaneous effects to interactions with the reservoir. However, the available data is too limited in extent and accuracy to conclusively show which of the models is the most physically correct. There are indications that the time factors used in the models are not constant but vary with the state of the system. It is interesting to note that as applied, both the ME and SEAQT approaches, along with the existing data, presume that entropy as described by the density matrix $\rho$ exists and can be measured for $a$ single particle. The data in Figure A2 in Appendix further supports this notion. The SEAQT point of view additionally claims that quantum systems that are not at stable thermal equilibrium can spontaneously increase in entropy, a claim that remains to be conclusively shown. SEAQT is also able to describe time lags that may exist, which the ME and DE models are unable to do. The discrepancies and similarities between the theoretical models will hopefully provide added rationale for further investigation, including new experiments to show the link between the entropy of the Second Law and Quantum Mechanics more clearly.

Acknowledgments: The author gratefully acknowledges Clinton L. Dancey for helpful discussions concerning this paper. 
Conflicts of Interest: The author claims no conflict of interest.

\section{Appendix: Comparison of the ME Model to Experimental Data}

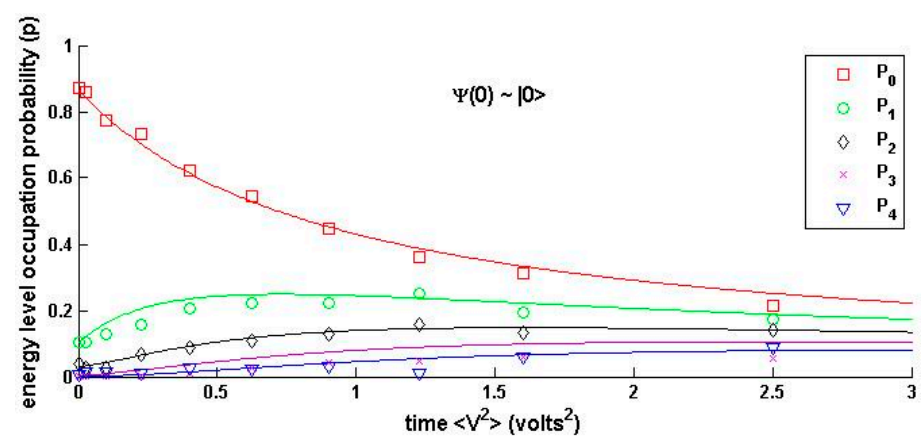

(a)

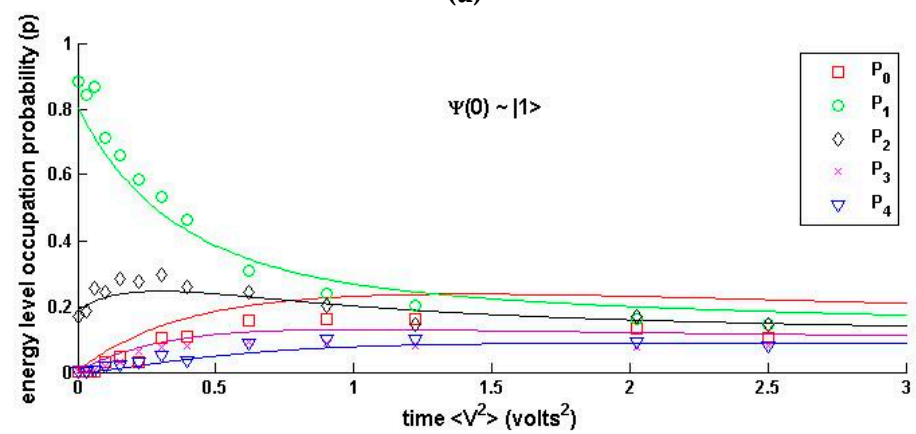

(b)

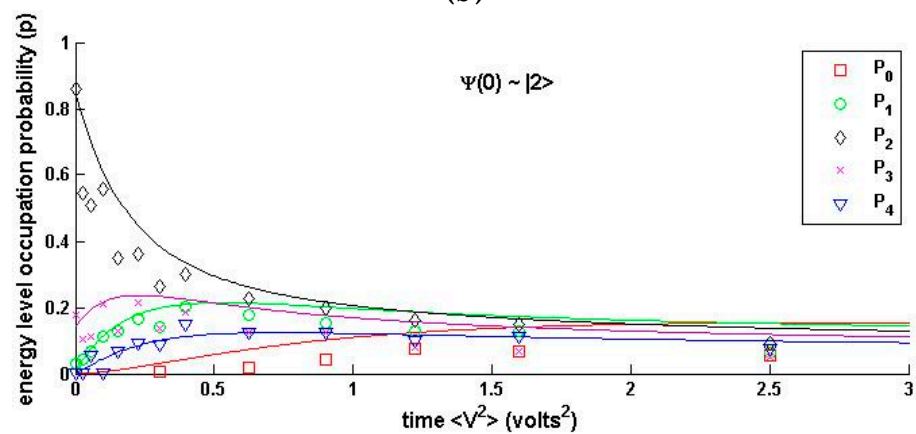

(c)

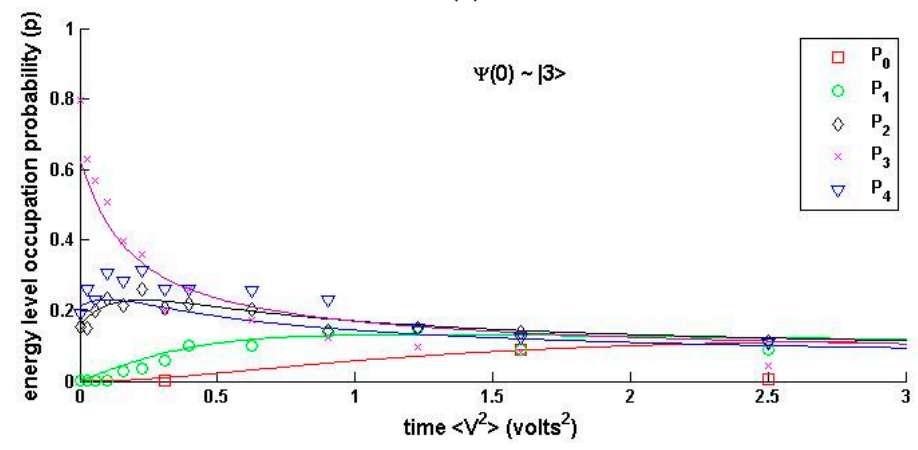

(d)

Figure A1. Comparison of the ME theoretical relaxation curves to the experimental data of [19] for initial states approximating $|0\rangle$ in $(\mathbf{a}),|1\rangle(\mathbf{b}),|2\rangle(\mathbf{c})$, and $|3\rangle$ (d) for the lowest five energy eigenlevels. The results are similar but not identical to [19]. A possible time lag is seen for the $\mathrm{P}_{0}$ data of the $|2\rangle$ (c) case. 


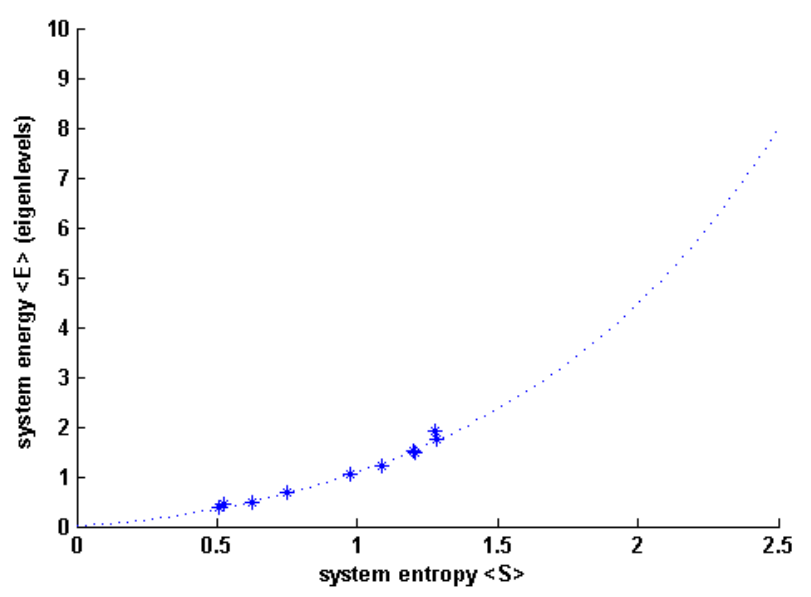

Figure A2. Energy vs. entropy evolution curve for experimental data from [19] for an initial state approximating the $10>$ eigenstate. The data follow but do not go to the right of the predicted limit curve of stable equilibrium states-physical evidence that single-particle quantum systems can exist in states with entropy greater than zero while obeying the limitations imposed by the Second Law.

\section{References}

1. Rauer, B.; Schweigler, T.; Langen, T.; Schmiedmayer, J. Does an isolated quantum system relax? 2015.

2. Stuart, C. The Quantum Thermodynamics Revolution. Available online: http://fqxi.org/community/ articles/display/202 (accessed on 6 May 2016).

3. Ladd, T.D.; Jeleszk, F.; Laflamme, R.; Nakamura, Y.; Monroe, C.; O’Brien, J.L. Quantum computers. Nature 2010, 464, 45-53. [CrossRef] [PubMed]

4. Cohen, A.J.; Mori-Sanchez, P.; Yang, W. Challenges for density functional theory. Chem. Rev. 2012, 112, 289-320. [CrossRef] [PubMed]

5. Jones, R.O. Density functional theory: Its origins, rise to prominence, and future. Rev. Mod. Phys. 2015, 87, 897-923. [CrossRef]

6. Weiss, U. Quantum Dissipative Systems; World Scientific: Singapore, Singapore, 2008.

7. Gisin, N. A simple nonlinear dissipative quatum evolution equation. J. Phys. A 1980, 14, $2259-2267$. [CrossRef]

8. Schuch, D. Nonunitary connection between explicitly time-dependent and nonlinear approaches for the description of dissipative quantum systems. Phys. Rev. A 1997, 55, 935. [CrossRef]

9. Blum, K. Density Matrix Theory and Applications; Springer: Berlin/Heidelberg, Germany, 2012.

10. Carmichael, H. An Open Systems Approach to Quantum Optics; Springer: Berlin/Heidelberg, Germany, 1991.

11. Hatsopoulos, G.N.; Gyftopoulos, E.P. A Unified Quantum Theory of Mechanics and Thermodynamics. Part I. Postulates. Found. Phys. 1976, 6, 15-31. [CrossRef]

12. Hatsopoulos, G.N.; Gyftopoulos, E.P. A Unified Quantum Theory of Mechanics and Thermodynamics. Part IIa. Available Energy. Found. Phys. 1976, 6, 127-141. [CrossRef]

13. Hatsopoulos, G.N.; Gyftopoulos, E.P. A Unified Quantum Theory of Mechanics and Thermodynamics. Part IIlb. Stable Equilibrium States. Found. Phys. 1976, 6, 439-455. [CrossRef]

14. Hatsopoulos, G.N.; Gyftopoulos, E.P. A Unified Quantum Theory of Mechanics and Thermodynamics. Part III. Irreducible Quantal Dispersions. Found. Phys. 1976, 6, 561-570. [CrossRef]

15. Beretta, G.P. On the General Equation of Motion of Quantum Thermodynamics and the Distinction between Quantal and Nonquantal Uncertainties. Sc.D. Thesis, Massachusetts Institute of Technology, Cambridge, MA, USA, 1981.

16. Beretta, G.P. What is Quantum Thermodynamics. Available online: http:/ /quantum-thermodynamics.unibs. it/WebSite1.pdf (accessed on 6 May 2016). 
17. Younis, A.M. Modeling the Non-Equilibrium Phenomenon of Diffusion in Closed and Open Systems at an Atomistic Level Using Steepest-Entropy-Ascent Quantum Thermodynamics. Ph.D. Thesis, Virginia Polytechnic Institute and State University, Blacksburg, VA, USA, 2015.

18. Leibfried, D.; Blatt, R.; Monroe, C.; Wineland, D. Quantum dynamics of single trapped ions. Rev. Mod. Phys. 2003, 75, 281-324. [CrossRef]

19. Turchette, Q.A.; Myatt, C.J.; King, B.E.; Sackett, C.A.; Kielpinski, D.; Itano, W.M.; Monroe, C.; Wineland, D.J. Decoherence and Decay of Motional Quantum States of a Trapped Atom Coupled to Engineered Reservoirs. Phys. Rev. A 2000, 62, 053807. [CrossRef]

20. Gring, M.; Kuhner, T.M.; Langen, T.; Kitagawa, T.; Rauer, B.; Schreitl, M.; Maxets, I.; Smith, D.A.; Demler, E.; Schmiedmayer, J. Relaxation and prethermalization in an isolated quantum system. Science 2012, 337, 1318-1322. [CrossRef] [PubMed]

21. Pefinova, V.; Luks, A. Exact quantum statistics of a nonlinear dissipative oscillator evolving from an arbitrary state. Phys. Rev. A 1990, 41, 414. [CrossRef]

22. Kim, M.S.; Buzek, V. Schrödinger-cat states at finite temperature: Influence of a finite-temperature heat bath on quantum interferences. Phys. Rev. A 1992, 46, 4239. [CrossRef] [PubMed]

23. Kreuzer, H.J. Nonequilibrium Thermodynamics and Its Statistical Foundations; Oxford University Press: Oxford, UK, 1981.

24. Beretta, G.P. Maximum Entropy Production Rate in Quantum Thermodynamics. J. Phys. Conf. Ser. 2010, 237, 012022. [CrossRef]

25. Beretta, G.P. A theorem on Lyapunov stability for dynamical systems and a conjecture on a property of entropy. J. Math. Phys. 1986, 27, 305. [CrossRef]

26. Beretta, G.P. Steepest-Entropy-Ascent Irreversible Relaxation Towards Thermodynamic Equilibrium: The Dynamical Ansatz that Completes the Gyftopoulos-Hatsopoulos Unified Theory with a General Quantal Law of Causal Evolution. Int. J. Thermodyn. 2006, 9, 117-128.

27. Beretta, G.P. Nonlinear Model Dynamics for Closed-System, Constrained, Maximal-Entropy-Generation Relaxation by Energy Redistribution. Phys. Rev. E 2006, 73, 026113. [CrossRef] [PubMed]

28. Li, G.; Al-Abbasi, O.; von Spakovsky, M.R. Atomistic-level non-equilibrium model for chemically reactive systems based on steepest entropy-ascent quantum thermodynamics. J. Phys. Conf. Ser. 2014, 538, 012013. [CrossRef]

29. Cano-Andrade, S.; Beretta, G.P.; von Spakovsky, M.R. Steepest-entropy-ascent quantum thermodynamic modeling of decoherence in two different microscopic composite systems. Phys. Rev. A 2015, 91, 013848. [CrossRef]

30. Hsiang, J.T.; Hu, B.L. Nonequilibrium steady state in open quantum systems: influence action, stochastic equation and power balance. 2014.

31. Smith, C.E. Intrinsic Quantum Thermodynamics: Application to Hydrogen Storage on a Carbon Nanotube and Theoretical Consideration of Non-Work Interactions. Ph.D. Thesis, Virginia Polytechnic Institute and State University, Blacksburg, VA, USA, 2012.

32. Nagel, M.; Haworth, F.E. Advanced Laboratory Experiments on Optical Pumping of Rubidium Atoms-Part II: Free Precession. Am. J. Phys. 1966, 34, 559. [CrossRef]

33. Kukolich, S.G. Time Dependence of Quantum-State Amplitudes Demonstrated by Free Precession of Spins. Am. J. Phys. 1968, 36, 420. [CrossRef]

34. Balabas, M.V.; Karaulanov, T.; Ledbetter, M.P.; Budker, D. Polarized Alkali Vapor with Minute-Long Transverse Spin-Relaxation Time. Phys. Rev. Lett. 2010, 105, 070801. [CrossRef] [PubMed]

35. Bollinger, J.J.; Heinzen, D.J.; Itano, W.M.; Gilbert, S.L.; Wineland, D.J. A 303-MHz frequency standard based on trapped Be/sup +/ ions. IEEE Trans. Instrum. Meas. 1991, 40, 126-128. [CrossRef]

36. Hatsopoulos, G.N.; Keenan, J.H. Principles of General Thermodynamics; Wiley: New York, NY, USA, 1965.

37. Gyftopoulos, E.P.; Beretta, G.P. Thermodynamics, Foundations and Applications; Dover: Mineola, NY, USA, 1991.

(C) 2016 by the author; licensee MDPI, Basel, Switzerland. This article is an open access article distributed under the terms and conditions of the Creative Commons Attribution (CC-BY) license (http://creativecommons.org/licenses/by/4.0/). 